\title{
A DEPRESSÃo GERIÁTRICA E SUA RELAÇÃO COM A SOLIDÃO NUMA AMOSTRA DE UTENTES COM SERVIÇO DE APOIO DOMICILIÁRIO
}

GERIATRIC DEPRESSION AND ITS RELATION WITH LONELINESS IN A SAMPLE OF SUBJECTS WITH HOME SUPPORT SERVICE

LA DEPRESIÓN GERIÁTRICA Y SU RELACIÓN CON LA SOLEDAD EN UNA

MUESTRA DE SUJETOS CON SERVICIO DE AYUDA A DOMICILIO

\author{
Ricardo Pocinho (pocinho@ hotmail.com)* \\ Tatiana Neves (tatiana.isabel.neves@gmail.com) \\ Pedro Belo (pedro.belo.santos@gmail.com) \\ Anabela Martins (anabelacmartins@estescoimbra.pt)
}

\begin{abstract}
RESUMO
O Serviço de Apoio Domiciliário (SAD) tem sido implementado na sociedade como um apoio social às pessoas idosas. Com caraterísticas de intervenção semelhantes ao Centro de Dia, o SAD caraterizase por uma deslocação ao domicílio de técnicos com a perspetiva de promover suporte básico diário ao idoso. Este estudo, realizado em Coimbra, teve como objetivo perceber se o Serviço de Apoio Domiciliário evita a Solidão e, consequentemente, a existência de sintomatologia depressiva. Metodologicamente foi estudado um modelo preditor da sintomatologia depressiva geriátrica, tendo por base a Satisfação com o Serviço de Apoio Domiciliário e a Solidão. Observou-se, através de um modelo ajustado, que a Solidão é um preditor significativo da Depressão Geriátrica (r2=0,607; $\mathrm{p}<, 05)$, havendo uma correlação significativa e positiva entre Solidão e Depressão Geriátrica $(\mathrm{r}=7,41 ; \mathrm{p}<, 05)$. Assim, associada à Solidão pode surgir a Depressão Geriátrica, a qual pode comprometer o funcionamento cognitivo do idoso que começa por manifestar dificuldades nos processos mais complexos, principiando por interferir na tomada de decisão, na autonomia e na qualidade de vida. Além de um Serviço de Apoio Domiciliário, deverá ser proporcionado ao idoso um suporte social que lhe permita preencher o vazio, evitando a solidão.
\end{abstract}

Palavras-chave: Serviço de Apoio Domiciliário, Idoso, Solidão, Depressão Geriátrica.

\begin{abstract}
The Home Support Service has been implemented in society as a social support to the elderly. With similar intervention characteristics of one Day Center, this is a technical home visit with the perspective of promoting daily basic support to the elderly. The aim of this study, conducted in Coimbra, is to perceive if the Home Support Service prevents solitude and, hence, the existence of depressive symptoms. We studied a predictor model of geriatric depression based on the satisfaction with the Home Support Service and on Loneliness. We observed through an adjusted model that Loneliness is a significant predictor of Geriatric Depression ( $\mathrm{r} 2=0,607 ; \mathrm{p}<, 05)$, and there is a positive correlation between Loneliness and Geriatric Depression $(r=7,41 ; \mathrm{p}<, 05)$. In association with
\end{abstract}




\title{
Egitania \\ s c i e $\Omega$ c i a
}

Loneliness comes the Geriatric Depression, which can compromise cognitive functioning of the elderly that begin to manifest difficulties in more complex processes starting to interfere in decision making, autonomy and quality of life. In addition to a Home Support Service, a social support should be provided to the elderly to enable them to fill the void avoiding solitude.

Keywords: Hom Keywords: Home Support Service, Elderly, Loneliness, Geriatric Depression.e Support Service, Elderly, Loneliness, Geriatric Depression.

\section{RESUMEN}

El servicio de ayuda a domicilio se ha implementado como un apoyo social a las personas séniores. Con características similares a la intervención de un centro de día, el SAD se caracteriza por un cambio de domicilio técnico con el fin de promover el apoyo básico diario a los ancianos viejos. Este estudio, hecho en Coimbra, tuvo como objetivo percibir si el servicio de ayuda a domicilio evita la soledad y por lo tanto la existencia de síntomas depresivos. Metodológicamente fue estudiado un modelo predictor de la sintomatología depresiva geriátrica teniendo por base la satisfacción con el servicio de ayuda a domicilio y la soledad. Se observó a través de un modelo de conjunto, que la soledad es un predictor significativo de depresión geriátrica $(\mathrm{r} 2=0,607 ; \mathrm{p}<, 05)$, con una correlación positiva y significativa entre la soledad y la depresión geriátrica $(r=7,41 ; \mathrm{p}<, 05)$. Por lo tanto, asociado con la soledad puede surgir la depresión geriátrica, que puede poner en peligro el funcionamiento cognitivo del sénior empezando a manifestar dificultades en los casos más complejos. Además de un servicio domiciliario, debe ser proporcionado a los ancianos apoyo social que les permite llenar el vacío, evitando la soledad.

Palabras clave: Servicios Apoyo a Domicilio, Senior, Soledad, Depresión Geriátrica.

\author{
*Doutor em Psicogerontologia (Universidade de Valência, Espanha). Membro do \\ Grupo de Investigação INTERTECH da Universidade de Valência (Espanha). \\ Reitor da Universidade Sénior do Mondego. \\ (http://www.degois.pt/visualizador/curriculum.jsp?key=2891052432293022Sub \\ mitted: 13th July 2015
}




\section{Egitania \\ $s$ c i e $\Omega$ c i a}

\section{INTRODUÇÃO}

Atualmente, o envelhecimento demográfico constitui, de forma geral, um problema para as sociedades e o seu desenvolvimento. $O$ índice de envelhecimento da população em Portugal é no ano de 2015 de 146,5\%, o que constitui uma aumento de 80,8\% comparando com o ano de 1990 (INE, 2016). Se o envelhecimento demográfico foi uma das grandes conquistas da humanidade, seguramente nos últimos dois séculos, este é também um dos grandes desafios, tanto a nível pessoal/individual, quanto a nível social/coletivo, sendo necessárias adaptações, não só às alterações biológicas e psicológicas dos indivíduos envelhecidos, mas também às que vão ocorrendo ao nível societal onde serão necessárias mudanças nas políticas sociais, culturais, éticas e económicas. As políticas sociais constituem parte do ramo da política de intervenção social que, através de vários instrumentos e meios, providenciam bens e serviços essenciais para garantir bem-estar, qualidade de vida, maior autonomia e independência, acesso e assistência aos cuidados de saúde para toda a população. No âmbito das políticas sociais para a velhice estão, entre outras, a Pensão Social de Velhice, Pensão de Sobrevivência, Pensão Social de Invalidez, Pensão de Viuvez e Complemento Solidário para os Idosos (Gomes \& Mata, 2012). Assim estas políticas são um conjunto de intervenções e ações sociais do Estado que têm o objetivo de responder às necessidades subjacentes à entrada nesta fase do ciclo vital (Gomes \& Mata, 2012; Pocinho, Jacob, Santos, \& Fernandes, 2013).

Com o aumento da idade da população, representada pelo crescimento da esperança de vida na quase totalidade dos países, torna-se pertinente encontrar outras formas de combater as necessidades inerentes ao envelhecimento. Cada pessoa tem as suas próprias necessidades e cada um sente-as de forma diferente. Neste sentido e, para fazer face às novas necessidades da população idosa, priorizando sua permanência no próprio domicílio, foi criado o Serviço de Apoio Domiciliário (SAD). Este surgiu com o objetivo de assegurar à população idosa e aos seus cuidadores a satisfação das necessidades básicas diárias; prestar apoio físico e psicossocial em contexto domiciliário, contribuindo, assim, para o aumento da qualidade de vida e do bem-estar dos idosos. Aparece no início dos anos 80, como uma política de prevenção e manutenção das pessoas em contexto domiciliário, permitindo que o idoso tenha uma maior qualidade de vida e permaneça em casa por mais tempo, ao invés da família o institucionalizar (Jacob, 2012). Emerge com o intuito de prestar os mesmos serviços do centro de dia (CD), só que em contexto domiciliário, tentando, de certa forma, evitar a exclusão social e a solidão (Jacob, 2012).

Segundo Oliveira (2008), a solidão na terceira idade não é tema de discussão constante, pois está relacionada, embora nem sempre diretamente, a outras variáveis. Este é um tema delicado, que pode ser abordado de várias perspetivas e que tem como origem vários fatores. São vários os estudos, por exemplo, que revelam que com as visitas de familiares e filhos, o idoso não apresenta menor exposição à solidão (Oliveira, 2008). A solidão é um risco eminente e presente no dia-a-dia dos sujeitos idosos em Portugal, pelo que é urgente criar estratégias que a minimizem. Esta diminui a auto-estima, o que, por sua vez, aumenta a prevalência de problemas físicos, mentais, sociais e de bem-estar (Belo, Pocinho, \& Costa, 2016). Todas estas mudanças condicionam o idoso e estão associadas à perda de autonomia, 


\section{Eqitania \\ s c i e $\Omega$ c i a}

ao sentimento de inutilidade, de culpa, de depressão, de desvalorização pessoal e de isolamento social.

Neste seguimento, o aumento das perdas e as perturbações biológicas, fatores relativamente comuns a esta faixa etária, estão habitualmente associados às perturbações depressivas (Pereira, Roncom, \& Carvalho, 2011). A depressão nas pessoas idosas é um fenómeno complexo que se manifesta não devido a uma causa isolada, mas a um conjunto de processos multi-fatoriais. Alguns dos investigadores referem que a prevalência da depressão se mantém constante ao longo do percurso de vida, contudo o crescente aumento de patologias e de limitações funcionais aumentam, também, os fatores de risco associados à mesma. Os principais fatores de risco identificados pelos investigadores são os fatores genéticos, psicológicos, ambientais, doenças crónicas, o luto, a solidão, o género (com maior prevalência entre as mulheres), dificuldades económicas e até a própria idade (Pereira, Roncom, \& Carvalho, 2011). A depressão manifesta-se através de vários sintomas, contudo os mais comuns são a apatia, a insónia, alterações de apetite, perda de auto-estima, isolamento social, sentimentos de desespero, bem como de abandono, e ainda uma visão negativa face a todo o meio envolvente (Vieira, 2004).

Os estudos indicam que as pessoas idosas com maior sintomatologia depressiva apresentam uma maior probabilidade de alterações e declínio cognitivo (Williamson, Shaffer, \& Parmelee, 2002). Assim, a depressão condiciona o funcionamento cognitivo que começa por manifestar dificuldades em processos mais complexos, começando por interferir na capacidade de tomada de decisão, autonomia e na qualidade de vida diária (Freeman et al., 2016). Assim, o diagnóstico da depressão apresenta-se, neste momento, como sendo um desafio, pois, devido às alterações cognitivas, é possível levar os profissionais de saúde a acreditarem estar perante um quadro demencial e não depressivo.

Neste contexto de perturbação psicológica, é imprescindível que exista um diagnóstico diferencial e multidisciplinar, utilizando exames físicos e testes psicométricos (Vieira, 2004). O estudo de Pereira et al. (2011) indica que os idosos que sofrem de solidão e de depressão têm um risco dobrado de mortalidade.

\section{MÉTODO}

O presente estudo, quasi-experimental, com amostragem por conveniência, pretende analisar a relação da Depressão e da Solidão numa amostra de pessoas idosas que usufruem de SAD. A recolha de informação foi realizada através de questionário na presença dos inquiridos, informando da sua confidencialidade e do tratamento global dos dados. Assim, fizeram parte da amostra de estudo apenas os utentes que concordaram com o Termo de Consentimento apresentado. Foi preenchido um questionário sociodemográfico e, em seguida, aplicada a Escala de Solidão - UCLA Loneliness Scale, uma das medidas mais usadas para avaliar a Solidão (Russell, Plepau, \& Ferguson, 1978; Russell, Plepau, \& Cutrona, 1990, versão portuguesa adaptada por Pocinho, Farate, \& Dias, 2010) e a Escala de Depressão Geriátrica - GDS (Pocinho, et al., 2009; Veríssimo, 1988; Yesavage, Brink, Rose, Lum, Huang, Adey, \& Leirer, 1983). 
O questionário sociodemográfico foi elaborado focando os aspetos que podem ter influência nas duas variáveis em estudo, a Solidão e a Depressão. Este permite-nos obter informações mais detalhadas sobre os dados pessoais, as relações interpessoais, contexto habitacional e a opinião referente à contratualização do Serviço de Apoio Domiciliário. A Escala de Solidão - UCLA, na versão portuguesa, é constituída por 16 itens e tem duas dimensões (isolamento social e afinidades), o que permite avaliar os sentimentos subjetivos de solidão e de isolamento social. A Escala de Depressão Geriátrica - GDS é formada por 30 questões de auto-resposta nas quais o indivíduo responde (Sim ou Não) conforme se tenha sentido na última semana. Esta permitiu-nos perceber a sintomatologia depressiva dos participantes. Os dados foram analisados recorrendo ao instrumento de análise estatística IBM-SPSS v. 21, assim como o software AMOS. Uma vez que o $n$ obtido foi inferior a 30 sujeitos (Pestana \& Gageiro, 2010), recorreu-se a estatística não-paramétrica para a análise de dados relacionais (ró spearman). Foi também usada a regressão linear múltipla e a regressão linear simples, no modelo ajustado obtido através do software AMOS.

\section{CARATERIZAÇÃO DA AMOSTRA}

A amostra foi composta por 10 indivíduos com idades acima dos 65 anos. Todos os participantes usufruem do Serviço de Apoio Domiciliário (SAD), de uma instituição de repouso localizada na zona centro de Portugal. Sendo um estudo de caso da referida instituição, teve-se o cuidado de retirar toda a informação que de alguma forma pudesse identificar os participantes.

Na amostra, 7 (70\%) participantes eram do género feminino e 3 (30\%) do género masculino (Cf. Tabela 1).

\begin{tabular}{lr}
\multicolumn{2}{c}{ Tabela 1 - Caraterização do grupo em relação ao género } \\
\hline Feminino & $70 \%(n=7)$ \\
Masculino & $30 \%(n=3)$ \\
Total & $100 \%(n=10)$ \\
\hline
\end{tabular}

Como se pode observar na Tabela 2, foram distribuídas as idades por faixas etárias havendo três utentes com idade compreendidas entre 65-74 anos; um tem idade compreendida entre 74-85 anos e os restantes (seis) têm idade igual ou superior a 85 anos de idade (60\%).

Tabela 2 - Caraterização do grupo em relação à faixa etária

\begin{tabular}{lr}
\hline Faixa Etária & Percentagem \\
\hline $\mathbf{6 5 - 7 4}$ & $30 \%(n=3)$ \\
$\mathbf{7 5 - 8 4}$ & $10 \%(n=1)$ \\
lgual $/>\mathbf{8 5}$ & $60 \%(n=6)$ \\
\hline
\end{tabular}

No que diz respeito às relações interpessoais, na pergunta "quem lhe presta apoio habitualmente?" (Cf. Tabela 3), 20\% dos participantes refere que são apoiados pela família e pelas instituições, $70 \%(n=7)$ diz receber apoio apenas de instituições e os restantes $10 \%$ são apoiados por instituições e pelos vizinhos, que têm aqui um papel preponderante. 
Tabela 3 - Caraterização de grupo quanto à prestação de cuidados

\begin{tabular}{lc}
\hline Quem Ihe presta apoio habitualmente? & Percentagem \\
\hline Familiares e Instituições & $20 \%(n=2)$ \\
Instituições & $70 \%(n=7)$ \\
Vizinhos e Instituições & $10 \%(n=1)$ \\
\hline
\end{tabular}

Relativamente à opinião dos participantes face à globalidade da prestação de serviços do Serviço de Apoio Domiciliário, 70\% encontra-se satisfeito, $20 \%$ muito satisfeito e os restantes $10 \%$ encontram-se pouco satisfeitos, tal como se observa na Tabela 4.

Tabela 4 - Grau de satisfação em relação ao SAD

\begin{tabular}{lc}
\hline $\begin{array}{l}\text { Grau de satisfação em relação à globalidade da prestação do } \\
\text { SAD }\end{array}$ & Percentagem \\
\hline Pouco satisfeito & $10 \%(n=1)$ \\
Satisfeito & $70 \%(n=7)$ \\
Muito Satisfeito & $20 \%(n=2)$ \\
Total & $100 \%$ \\
\hline
\end{tabular}

Todos os sujeitos da amostra são de opinião que este serviço veio minorar o sentimento de solidão que sentiam no seu quotidiano (Cf. Tabela 5).

Tabela 5 - Perceção do grupo quanto ao contributo do SAD para minorar o sentimento "solidão"

\begin{tabular}{lr}
\hline O serviço contribuiu para minorar o sentimento de & Percentagem \\
tão? & $100 \%$ \\
\hline Sim & $10 \%$
\end{tabular}

\section{RESULTADOS}

Podemos observar na Tabela 6 que a pontuação média da Escala de Solidão (UCLA) da amostra é de 47,80 (dp=7,51), com uma pontuação total mínima de 38 e uma pontuação máxima de 57. Por conseguinte, segundo a pontuação global (normativa) para a existência de solidão (média> 32), podemos dizer que os participantes do estudo apresentam sentimento de solidão (média amostra $=47,8>$ média normativa $=32$ ).

Tabela 6 - Caraterização da amostra em relação à pontuação total UCLA

\begin{tabular}{lllll}
\hline & Média & Desvio padrão & Mínima & Máxima \\
\hline UCLA & 47,80 & 7,510 & 38 & 57
\end{tabular}

Analisando as sub-escalas da UCLA na Tabela 7, podemos observar que a amostra em estudo pontua mais na dimensão Isolamento Social (média $=2,04 ; \mathrm{dp}=, 458$ ) do que na dimensão Afinidades (média $=1,92 ; \mathrm{dp}=, 738$ ). 
Tabela 7 - Caraterização da amostra em relação à pontuação total UCLA

\begin{tabular}{lcccc}
\hline UCLA & Média & Desvio padrão & Mínima & Máxima \\
\hline Isolamento Social & 2,04 &, 458 & 1 & 3 \\
\hline Afinidades & 1,92 &, 738 & 1 & 3 \\
\hline
\end{tabular}

Relativamente à Escala de Depressão Geriátrica (DGS) os resultados obtidos mostram que a pontuação média é de 18 ( $d p=5,011)$. Segundo Pocinho et al. (2009) uma pontuação de onze a vinte, é considerada "depressão leve", pelo que podemos concluir que os participantes da amostra se sentem levemente deprimidos.

Tabela 8 - Caraterização da amostra em relação à pontuação total DGS

\begin{tabular}{lcccc}
\hline & Média & Desvio padrão & Mínima & Máxima \\
\hline DGS & 18,00 & 5,011 & 10 & 27 \\
\hline
\end{tabular}

Para estudar a associação que se estabelece entre as variáveis e, uma vez que o valor de $n$ é inferior a 30 sujeitos, foi efectuado o teste do ró de Spearman (Pestana \& Gageiro, 2010). Analisando a Tabela 9, podemos observar que ocorre uma relação estatisticamente significativa e positiva entre a Solidão (UCLA-Total) e a Depressão (GDS-Total), sendo que, quando a Solidão aumenta, a sintomatologia depressiva também aumenta nos sujeitos da $\operatorname{amostra}(\mathrm{p}<.05)$.

\begin{tabular}{|c|c|c|}
\hline & \multicolumn{2}{|c|}{ Solidão (UCLA-Total) } \\
\hline & $R$ & $P$ \\
\hline Depressão & & \\
\hline (DGS-Total) & ,741 &, $014 *$ \\
\hline
\end{tabular}

De seguida, é apresentado na Tabela 10, o modelo preditivo da Depressão Geriátrica, constituído pelas variáveis Solidão e Grau de Satisfação com o SAD. Os resultados mostram que o modelo preditivo estudado explica $60,7 \%$ da Depressão Geriátrica na amostra.

Tabela 10 - Modelo preditivo da Depressão

\begin{tabular}{|c|c|c|c|c|c|c|}
\hline \multicolumn{7}{|c|}{ GDS-Total } \\
\hline & $r^{2}$ & $r^{2} a j$. & $\mathrm{P}$ & B & Beta & $\mathrm{T}$ \\
\hline \multicolumn{7}{|l|}{ UCLA-Total } \\
\hline & & & & 479 & ,717 & $3,01 *$ \\
\hline \multirow[t]{2}{*}{ Grau de Satisfação SAD } & 607 & 495 &, $038^{*}$ & & & \\
\hline & & & & $-2,13$ & -241 & $-1,04$ \\
\hline
\end{tabular}

A significância do efeito do Grau de Satisfação e da Solidão sobre a Depressão Geriátrica foi avaliada com uma regressão linear múltipla com estimação dos parâmetros pelo método da máxima verosimilhança implementado no software AMOS. Analisando o modelo ajustado à 
Depressão Geriátrica em função do Grau de Satisfação com o SAD e a Solidão, podemos concluir que apenas a Solidão é um preditor significativo da Depressão Geriátrica (Figura 1).

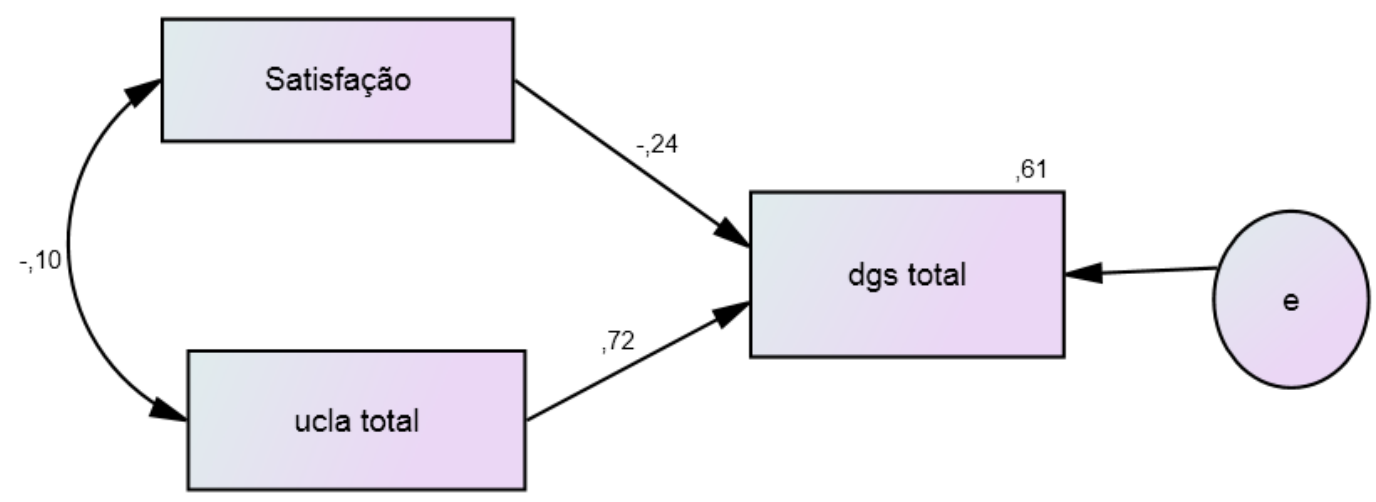

$\mathrm{CFI}=1,000 ; \mathrm{PCFI}=, 000 ; \mathrm{NFI}=1,000 ; \mathrm{NCP}=, 000 ; \mathrm{RMSEA}(p)=, 451(, 040)$

Figura 1. Modelo de regressão linear múltipla da Depressão Geriátrica em função da Satisfação com o $S A D$ e a Solidão $\left(r^{2}=0.61\right)$.

A Figura 1 apresenta o modelo com as estimativas estandardizadas, onde não se observa um efeito significativo para o Grau de Satisfação com o SAD (Beta Dep_Sat= -2.41; p=0.344).

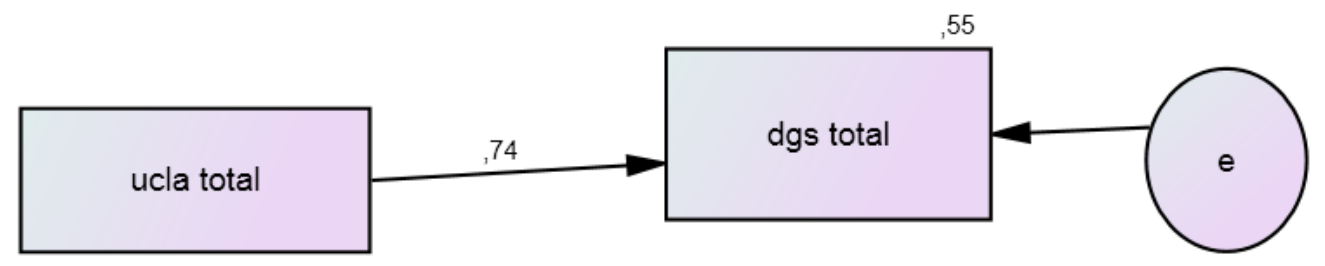

Figura 2. Modelo de regressão linear simples entre a Depressão Geriátrica em função da Solidão $\left(r^{2}=0.55\right)$.

A existência de uma multicolinearidade forte entre a Solidão e o Grau de Satisfação $(\mathrm{VIF}=1,010)$ recomenda a remoção de uma das variáveis passando a ser considerada apenas a trajetória significativa. O refinamento resultou no modelo da predição (regressão linear simples) da Depressão Geriátrica apresentado na Figura 2.

\section{DISCUSSÃO}

Este estudo permitiu o desenho de um modelo estrutural sobre a influência da solidão no estado depressivo dos idosos com apoio domiciliário. A maioria dos idosos recorre a este tipo de serviço social quando já se sente incapaz de realizar determinadas tarefas e não possui, ou não pode recorrer a outra rede de apoio. Assim, o contato com o Serviço de Apoio Domiciliário (SAD) é incentivado pela rede de suporte informal, quando os idosos 


\section{Eqitania \\ s c i e $\Omega$ c i a}

apresentam já limitações ao nível físico e/ou ao nível cognitivo. A opinião dos inquiridos em relação à prestação do Serviço de Apoio Domiciliário é a de que se encontram satisfeitos (70\%). Quanto à importância do Serviço de Apoio Domiciliário para minimizar o sentimento de solidão e no aumento do bem-estar e qualidade de vida, todos referiram esta ser uma resposta preponderante nas suas vidas. Contudo, os resultados mostram que deveria haver mais apoio social, com a criação, ampliação e fortalecimento de outras redes de suporte, à semelhança do que refere Beyer, et al. (2015). Apesar de haver uma manifestação latente da satisfação com um serviço de apoio domiciliário, é um fato que este serviço não é suficiente para evitar uma sintomatologia depressiva, sendo que a Solidão tende a levar a um aumento da Depressão Geriátrica, tal como se observa no estudo de McKee e Schuz (2015). Observando o modelo preditor estudado, é a Solidão que melhor explica a Depressão Geriátrica (55\%). Este resultado vem ao encontro dos estudos de Wurm e Benyamini (2014) que obtiveram resultados idênticos. O SAD possibilita um bem-estar momentâneo, contudo não é suficiente para evitar o sentimento de solidão e, consequentemente, uma sintomatologia depressiva nestes utentes. A solidão deverá ser combatida através de um apoio diário, havendo um maior envolvimento da rede de suporte social (familiares, amigos, comunidade), como refere Domingues (2012).

\section{CONCLUSÕES}

Sabemos que a população está cada vez mais envelhecida sendo que, associado ao fator idade, encontra-se, muitas vezes, o fator dependência. Apesar de muitas pessoas idosas não terem o apoio familiar desejado, a rede de suporte social continua a ser imprescindível para a promoção da saúde dos idosos.

Todos estes fatores, por vezes isolados, perfazem um conjunto de situações que leva a que o idoso se sinta isolado do mundo e até descaraterizado. Associada à Solidão pode surgir a Depressão, a qual pode comprometer o funcionamento cognitivo que começa por manifestar dificuldades nos processos mais complexos, começando por interferir na tomada de decisão, autonomia e na qualidade de vida. A fraca socialização dos idosos, em resultado da debilitação física para sair à rua, leva a um isolamento social, fazendo com que percam a sua identidade ao longo do tempo.

A análise global dos resultados nesta pequena amostra leva-nos a concluir que os utentes que experienciam solidão tendem a desenvolver uma sintomatologia depressiva. $\mathrm{O}$ apoio deve passar igualmente por um acompanhamento social adicional de modo a que o sentimento de solidão seja ultrapassado e a sintomatologia depressiva seja atenuada. Defendemos que se comece a dar mais atenção aos sujeitos idosos, através de redes de cuidado, bem como de apoio intergeracional, alargadas e aprofundadas a partir de uma compreensão da velhice como um processo natural, ainda que plural e diversificado, e pelo qual todos esperamos passar, e não como um castigo ou punição indesejável que acomete só aos fracos, vulnerabilizando-os ainda mais. Além disso, é muito importante que se valorize a sintomatologia apresentada, considerando imprescindível o envolvimento multidisciplinar no diagnóstico e tratamento das diferentes patologias inerentes ao envelhecimento e ao modo de vida. Sugerimos estudos 


\section{Egitania}

s c i e $\Omega$ c i a

futuros nesta área com mais participantes, uma vez que a presente amostra sendo pouco representativa, não permite avaliar com exatidão os dados da amostragem, os quais não são projetáveis na população.

\section{BIBLIOGRAFIA}

Belo, P., Pocinho, R., \& Costa, J. (2016). Análise das diferenças de GÉNero em termos de ansiedade numa amostra. In R. Pocinho, P. Belo, \& V. Anjos (Eds.), Conversas de Psicologia e do Envelhecimento Ativo - 2016 - Atas do Congresso (pP. 15 - 22). Coimbra : AssociaÇão Portuguesa Conversas de Psicologia.

Beyer, A., Wolff, J., Warner, L., SchüZ, B., \& Wurm, S. (2015). The role of physical activity in the RELATIONSHIP BETWEEN SELF-PERCEPTIONS OF AGEING AND SELF-RATED HEALTH IN OLDER ADULTS. PSYCHOLOGY \& HEALTH, 30(6), 671-685. HTTP://DX.DOI.ORG/10.1080/08870446.2015.1014370

DOMINGUeS, M. (2012). MAPA MÍNIMO DE RELAÇÕES DO IDOSO: UMA FERRAMENTA PARA AVALIAR REDE DE SUPORTE Social. In F. Pereira (Org.), TeOria e prática da Gerontologia: Um Guia Para Cuidadores de Idosos (PP. 175188). VISEU: PSICOSOMA.

Freeman, A., Santini, Z., Tyrovolas, S., Rummel-Kluge, C., Haro, J., \& Koyanagi, A. (2016). Negative PERCEPTIONS OF AGEING PREDICT THE ONSET AND PERSISTENCE OF DEPRESSION AND ANXIETY: FINDINGS FROM A PROSPECTIVE ANALYSIS OF THE IRISH LONGITUDINAL STUDY ON AGEING (TILDA). JOURNAL OF AFFECTIVE DiSORDERS, 199, 132 - 138. HTTP://DX.DOI.ORG/10.1016/J.JAD.2016.03.042

Gomes, M. J. \& Mata, M. (2012). Família provedora de cuidados ao idoso dependente. In F. Pereira (Org.), TeOria e Prática da Gerontologia: Um Guia PARA Cuidadores de Idosos (PP.163-174). Viseu: Psicosoma.

InSTITUTO NACIONAL DE EsTATÍSTICA-INE (2016). EstatísTICAS DEMOGRÁFICAS - 2015. LisboA: InSTITUTO NACIONAL DE EsTATÍSTICA.

Jacob, L. (2012). Respostas sociais para idosos em Portugal. In F. Pereira (Org.), Teoria e prática da Gerontologia: Um Guia Para Cuidadores de Idosos (PP.129-147). Viseu: Psicosoma.

McKee, K., \& SchuZ, B. (2015). Psychosocial factors in healthy ageing. Psychology \& Health, 30(6), 607626. HTTP://DX.DOI.ORG/10.1080/08870446.2015.1026905

Oliveira, J. H. B. (2008). PSicologia do idOSO, temas COMPLEMENTARES. PORTO: LiVPSiC, 2008.

Pereira, M., Roncom, J., \& CARVAlho, H. (2011). Aspetos PSicolóGicos E FAmiliares do ENVElhecimento. In Z. AZEREDo (ORG.), O IDOSO COMO UM TODO (PP.101-130). VisEU: PSICOSOMA.

Pestana, M., \& Gageiro, J. (2010). ANÁlise de dados Para CiÊNCIAS SOCIAis: a COMPlementaridade do SPSS. LISBOA: SÍLABO.

Pocinho, R., Jacob, L., SAntos, E., \& Fernandes, H. (2013). EnVelhecimento e Economia Social: Perspetivas ATUAIS. VISEU: PSICOSOMA

Pocinho, M., Farate, C., \& Dias, C. A. (2010). Validação Psicométrica da Escala UCla - Loneliness para Idosos Portugueses. INTERAÇõEs, 1(18), 65-77.

Pocinho, M., Farate, C., Dias, C. A., Lee, T., \& Yesavage, J. (2009). Clinical and Psychometric Validation of the Geriatric Depression Scale (GDS) for Portuguese Elders. Clinical Gerontologist, 2(32), 223-236. HTTP://DX.DOI.ORG/10.1080/07317110802678680

Russell, D., Peplau, L. A., \& Cutrona, C. (1990). The Revised UCla loneliness Scale: Concurrent and Discriminent Validity Evidence. Journal of Personality and Social Psychology, 39, 472-480.

Russell, D., Peplau, L. A., \& Ferguson, M. L. (1978). Developing a measure of loneliness. Journal of Personality Assessment, 42, 290-294.

VERÍSSIMO, M. T. (1988). AVALIAÇÃo DiAgNÓSTICA DAS SÍNDROMES DEMENCIAIS: ESCALA DE DEPRESSÃo GERIÁTRICA. Porto: Serviço de Neurologia do Hospital S. JoÃo do Porto.

Vieira, E. B. (2004). Manual de Gerontologia: Um Guia Teórico-Prático para Profissionais, Cuidadores e FAMILIARES. RIO DE JANEIRO: REVINTER.

Williamson, G.M., ShafFer, D.R., \& PARMeleE, P.A. (2002). Physical illness AND DEPRESSiOn IN Older adults. NeW York:KLuWer ACAdemic/Plenum Publishers.

WURM, S., \& BENYAMINI, Y. (2014). OPTIMISM BUFFERS THE DETRIMENTAL EFFECT OF NEGATIVE SELFPERCEPTIONS OF AGEING ON PHYSICAL AND MENTAL HEALTh. Psychology \& HeAlth. 29(7), 832-848. HTTP://DX.DOI.ORG/10.1080/08870446.2014.891737 


\section{Eqitania}

s c i e $\Omega$ c i a

Yesavage, J., Brink, T., Rose, T. L., Lum, O., Huang, V., Adey, M., \& Leirer, V. O. (1983). Development And VALIDATION OF A GERIATRIC DEPRESSION SCREENING SCALE: A PRELIMINARY REPORT. JOURNAL OF PSYCHIATRIC RESEARCH, 17, 37-49. 